\title{
-NOTES-
}

\section{FORMULATION OF BALANCE OF FORCES IN MIXTURE THEORIES*}

\author{
By M. L. OLIVER AND W. O. WILLIAMS (Carnegie-Mellon University)
}

1. Introduction. The fundamental concept of the continuum theory of mixtures, that of modeling the mixture as a collection of distinct continuous bodies coexistent in space, is both clear and intuitively pleasing. However, it is not a simple matter to decide what form the corresponding equations of balance should take. In [1] Oliver introduced an axiomatic derivation of balance laws for mixtures of rigid continua; in [2] Williams extended the axioms to motions of non-diffusive mixtures. It was hoped that such derivations might serve to give a more substantial base to the classical field theories for mixtures, but in fact the resulting equations differ significantly from the usual ones.

In this paper we illustrate these distinctions by considering the equation of balance of forces for a non-diffusive mixture. We present the classical formulation, then the formulation of Oliver and Williams and finally a related but different formulation which has been suggested in a note by Gurtin, Oliver and Williams [3].

2. Geometry, definitions. Let $\varepsilon$ denote three-dimensional euclidean space, and $B$ denote a smooth regular open region in $\varepsilon$. We call $B$ the body. Let $\mathbf{B}$ denote the collection of all smooth regular open regions contained in $B$. Each element $A$ in $\mathbf{B}$ is called a subbody of $B$.

We define operations $\vee$ and $\wedge$ on $\mathbf{B}^{2}=\mathbf{B} \times \mathbf{B}$ and ${ }^{*}$ on $\mathbf{B}$ as follows:

$$
A \vee C=A \cup C, \quad A \wedge C=\overline{A \cap} \bar{\cap}, \quad A^{*}=\bar{\circ} \overline{B / A}
$$

We say that elements $A, C \in \mathrm{B}$ are separate if $A \wedge C=\varnothing$, and we let sep $\mathbf{B}^{2}$ denote the collection of separate pairs $(A, C)$ in $\mathbf{B}^{2}$.

By a mixture we mean a body $B$ together with its collection $\mathbf{B}$ of subbodies and an index set $I=\{1,2, \cdots, n\}$ whose elements are called constituents. Physically, of course, we picture the mixture as a set of spatially coincident bodies within the region $B$. For each constituent $i$ in $I$ and element $A$ in $\mathbf{B}, A_{i}$ shall denote the part of constituent $i$ occupying the region $A$ in $B$. We shall refer to $B_{i}$ as the $i$ th constituent body.

Let $\mathcal{V}$ designate the translation space of $\mathcal{E}$. We define a force system for a non-diffusive mixture to be a collection of functions $F_{i j}, F_{i}{ }^{e}, i, j \in I$ with the following properties:

$$
\begin{gathered}
F_{i j}: \mathrm{B}^{2} \rightarrow \mathcal{V}, \quad i \neq j \\
F_{i i}: \operatorname{sep} \mathrm{B}^{2} \rightarrow \mathcal{V}, \quad F_{i}{ }^{e}: \mathbf{B} \rightarrow \mathcal{V} ;
\end{gathered}
$$

* Received May 8, 1973; revised version received November 30, 1973. 


$$
\begin{aligned}
& F_{i j} \text { is biadditive; }{ }^{1} \\
& F_{i}{ }^{e} \text { is additive. }
\end{aligned}
$$

For $(A, C) \in \mathbf{B}^{2}, F_{i j}(A, C)$ is the force exerted by $C_{i}$ on $A_{i} ;$ for $A \in \mathbf{B}, F_{i}{ }^{e}(A)$ is the force exerted on $A$, by the environment of $B$ (including inertial forces).

Given a force system for a mixture, we define for each $i \in I$ a new function $F_{i I}: \mathrm{B}^{2} \rightarrow \mathcal{V}$ as follows:

$$
F_{i I}(A, C)=\sum_{\substack{i \in I \\ j \neq i}} F_{i j}(A, C)+F_{i i}(A, \overline{C-A})+F_{i i}(\overline{A-0}-C, A \wedge C) .
$$

Note that (1) is defined even when $A \wedge C \neq \varnothing$. For $(A, C) \in \mathbf{B}^{2}$, we call $F_{\imath I}(A, C)$ the force exerted on $A_{i}$ by the mixture within $C$.

If forces are prescribed on parts of $\partial B$, we suppose that they are prescribed for each constituent; that is, if $\delta \subset \partial B$.

$$
f_{i}: s \rightarrow \mathcal{V}
$$

is given as the force on $\delta$ which must be supported by the $i$ th constituent.

3. Balance of forces in classical mixture theory. In classical mixture theory the law of balance of forces takes the form

$$
F_{i I}(A, B)+F_{i}{ }^{e}(A)=0 \text { for all } i \in I, A \in \mathbf{B} .
$$

This requires that the force exerted on $A_{i}$ by the mixture plus the force exerted on $A_{i}$ by the environment of $B$ must be zero. The forces in $(i)_{3}$ and (1) are presumed to be represented as follows:

$$
F_{i I}(A, C)=\int_{\partial A \cap \partial C} T_{i} n d a,
$$

for every $(A, C) \in \operatorname{sep} \mathbf{B}^{2}$, where $n$ is the unit normal to $\partial A$;

$$
F_{i I}(A, C)=\int_{A} p_{i} d v+\int_{\partial A \cap C} T_{i} n d a
$$

for every $(A, C) \in \mathrm{B}^{2}$ with $A \subseteq C$;

$$
F_{i}{ }^{e}(A)=\int_{A} b_{i}{ }^{e} d v+\int_{\partial A \cap \partial B} T_{i} n d a,
$$

for every $A \in \mathrm{B}$. Here $T_{i}, b_{i}{ }^{e}$ and $p_{i}$ are respectively $\mathscr{L}(\mathcal{V}), \mathcal{V}, \mathcal{V}$-valued functions on $B$ with the following interpretations: $T_{i}$ is the partial stress tensor for constituent $i, b_{i}{ }^{e}$ the external body force on constituent $i$, and $p_{i}$ the total diffusive force exerted on constituent $i$. Using (3)-(5), we can write (2) in the following integral form:

$$
\int_{\partial \boldsymbol{A} \cap B} T_{i} n d a+\int_{A} p_{i} d v+\int_{A} b_{i}{ }^{e} d v+\int_{\partial A \cap \partial B} T_{i} n d a=0,
$$

${ }^{1}$ A function $F: \mathbf{B} \rightarrow \mathcal{V}$ is additive if $F(A \vee C)=F(A)+F(C)$ whenever $A, C$, and $A \vee C$ all belong to $\mathbf{B}$ and $(A, C) \in \operatorname{sep} \mathbf{B}^{2}$. A function $F: \mathbf{B} \rightarrow \mathcal{V}$ is biadditive if it is additive in each argument, i.e., if $F(A \vee C, D)=F(A, D)+F(C, D)$ and $F(A, D \vee E)=F(A, D)+F(A, E)$ whenever $A, C, D$, $E, A \vee C$ and $D \vee E$ all belong to $\mathbf{B}$ and $(A, C),(D, E) \in \operatorname{sep} \mathbf{B}^{2}$. 
for every $A \in \mathbf{B}$. Locally this last equation reduces to

$$
\operatorname{div} T_{i}+p_{i}+b_{i}{ }^{e}=0 \text { for all } i \in I \text {. }
$$

Defining $^{2} T=\sum_{i \in I} T_{i}$ as the global stress tensor and using (6), we obtain

$$
\operatorname{div} T+\sum_{i \in I}\left(p_{i}+b_{i}{ }^{e}\right)=0 .
$$

In order to guarantee balance of forces for the mixture as a whole, the classical theory requires that

$$
\int_{\partial \boldsymbol{A}} T n d a+\int_{\boldsymbol{A}}\left(\sum_{i \in I} b_{i}{ }^{e}\right) d v=0
$$

for every $A \in \mathrm{B}$; locally this reduces to

$$
\operatorname{div} T+\sum_{i \in I} b_{i}{ }^{e}=0 .
$$

Eqs. (7) and (8) require

$$
\sum_{i \in I} p_{i}=0 .
$$

If forces $f_{i}$ be prescribed on $\varsigma \subset \partial B$, then the boundary conditions for $T_{i}$ are

$$
\left.T_{i} n\right|_{s}=f_{i} .
$$

Eqs. (6) and (9), together with the boundary conditions (10), are the equations of force balance for the classical theory.

4. Balance of forces in the OW theory. The axiomatic approaches to the theory of mixtures proposed by Oliver [1] and Williams [2] impose more stringent requirements on the system. Balance of forces for $A_{i}$ alone is ensured by (2) as in the classical theory. But, in addition, the requirement of balance of forces for such compound elements as $A_{i} \cup A_{i}$ leads to the following set of balance equations:

$$
F_{i I}(A, B)+F_{i}{ }^{e}(A)=0
$$

for all $i \in I, A \in \mathbf{B}$, and

$$
F_{i j}(A, C)+F_{i i}(C, A)=0
$$

for all $i, j \in I, i \neq j$, all $(A, C) \in \mathbf{B}^{2}$. Eq. (11) is also required to hold for $i=j$ provided that $(A, C) \in \operatorname{sep~} \mathbf{B}^{2}$. Moreover, the axiomatic scheme yields the following representations:

$$
F_{i j}(A, C)=\int_{\partial A \cap \partial C} \sigma_{i i}{ }^{\partial A} d a
$$

for every $(A, C) \in \operatorname{sep} \mathbf{B}^{2}$ and all $i, j \in I$;

$$
F_{i j}(A, C)=\int_{A} p_{i i} d v+\int_{\partial A \cap \partial C} \tau_{i j}{ }^{\partial A} d a,
$$

${ }^{2}$ For simplicity, we assume that the diffusive velocities are zero; the equation of balance for the mixture as a whole is much more complicated in the general case. 
for every $(A, C) \in \mathbf{B}^{2}$ with $A \subseteq C$ and for all $i, j \in I$ with $i \neq j$;

$$
F_{i}{ }^{e}(A)=\int_{A} b_{i}{ }^{e} d v+\int_{\partial A \cap \partial B} f_{i} d a
$$

for every $A \in \mathbf{B}$ and $i \in I$. Here ${\sigma_{i j}}^{s}$ and $\tau_{i j}{ }^{s}$ are defined for any smooth manifold $\delta$ in $B$ and map $\delta$ into $V_{;} f_{i}$ maps $\partial B$ into $\mathcal{V}_{;} p_{i j}$ and $b_{i}{ }^{e}$ map $B$ into $\mathcal{V}$. Here $\sigma_{i j}{ }^{\partial A}$ is the density of the force exerted on $A_{i}$ by $C_{i}$ through the surface of contact $\partial A \cap \partial C ;{\tau_{i j}}^{\partial A}$ is the density of the force exerted on $A_{i}$ by $A_{i}$ through the surface $\partial A ; f_{i}$ is the density of the force exerted on $B_{i}$ by the environment of $B$ across the surface of contact $\partial B ; p_{i j}$ is the density of the diffusive force exerted by $B_{i}$ on $B_{i}$, and $b_{i}{ }^{e}$ is as before.

In addition the axiomatic scheme yields for all $i, j \in I$

$$
\tau_{i j}{ }^{s}=-\sigma_{i j}{ }^{s} \quad(i \neq j),
$$

for every smooth manifold $\delta$ in $\stackrel{\circ}{\text {. By }}$ Bying Eqs. (12)-(15) the balance Eq. (2) becomes

$$
\int_{\partial \boldsymbol{A}}{\sigma{ }^{\partial i}{ }^{\partial A}} d a+\int_{A}\left(p_{i}+b_{i}{ }^{e}\right) d v=0,
$$

for every $A \in \mathbf{B}$ with $\partial A \cap \partial B$ of zero area and for every $i \in I$; here we have set $p_{2}=$ $\sum_{i \in I, i \neq i} p_{i j}$. The balance equation (11) becomes

$$
-\int_{\partial \boldsymbol{A}}\left(\sigma_{i j}{ }^{\partial A}+{\sigma_{i i}}^{\partial A}\right) d a+\int_{\boldsymbol{A}}\left(p_{i i}+p_{i i}\right) d v=0,
$$

for every $A \in \mathrm{B}$ and all $i, j \in I$ with $i \neq j$; and

$$
\int_{s}\left({\sigma_{i j}}^{\delta}+{\sigma_{i i}}^{-\delta}\right) d a=0,
$$

for every $S \subset \stackrel{\circ}{\mathrm{B}}$ and all $i, j \in I$. It follows from (15)-(18) that there exist mappings $T_{i j}$ from $B$ into $\mathscr{L}(\mathcal{V})$ such that

$$
\begin{aligned}
{\sigma_{i i}}^{s} & =T_{i i} n, \\
{\sigma_{i j}}^{s}+{\sigma_{i i}}^{s} & =T_{i j} n \quad(i \neq j),
\end{aligned}
$$

for every smooth manifold $S$ in $B$, where $n$ is the positive unit normal to $\delta . T_{i j}$ is the $i j$ stress tensor. The local forms of (16), (17), and (18) are

$$
\begin{aligned}
\operatorname{div} T_{i i}+p_{i}+b_{i}{ }^{e} & =0, & \\
\operatorname{div} T_{i j}-p_{i j}-p_{i i} & =0, & i \neq j, \\
{\sigma_{i i}}^{s}+\sigma_{i i}{ }^{-} & =0, & i \neq j .
\end{aligned}
$$

The complete set of local balance equations is then given by (19). If forces $f_{i}$ be prescribed on $\delta \subset \partial B$ for the $i$ th constituent, then boundary conditions for this system are

$$
\begin{aligned}
\left.T_{i i} n\right|_{s}-\sum_{\substack{i \in I \\
i \neq i}} \tau_{i j}{ }^{s}=f_{i}, \\
\left.T_{i j} n\right|_{\delta}+\tau_{i j}{ }^{s}+\tau_{j i}{ }^{s}=0 .
\end{aligned}
$$

The latter can also be written, using (15), as

$$
\left.T_{i i} n\right|_{\delta}-{\sigma_{i i}}^{s}-{\sigma_{i j}}^{\delta}=0 .
$$


By construction there are no further conditions necessary in order to guarantee balance of forces for the entire mixture.

If we define $T_{i}=\sum_{j \in I} T_{i i}$ and $\hat{p}_{i}=-\sum_{j \in I, i \neq i} p_{i i}$, summing (19) $)_{2}$ over $j \neq i$ and adding the result to (19) ${ }_{1}$ yields

$$
\operatorname{div} T_{i}+\hat{p}_{i}+b_{i}{ }^{e}=0,
$$

which is exactly of the form (6). However, the boundary conditions for $T_{i}$ are, using (20),

$$
\left.T_{i} n\right|_{s}+\sum_{\substack{i \in I \\ i \neq i}} \tau_{i i}{ }^{s}=f_{i},
$$

which differs from (10). Recapitulating, for this system, the basic equations are (19) with boundary conditions (20); as a consequence of these we obtain the balance equation (21) with boundary condition (22) (of course (21), (22) do not suffice to guarantee (19), (20)). As a consequence of the above, we have for the global stress tensor, defined to be

$$
T=\sum_{\substack{i, i \in I \\ i<i}} T_{i j}
$$

the equation

$$
\operatorname{div} T+\sum_{\substack{i \in I \\ j \neq i}}\left(p_{i}+b_{i}{ }^{e}\right)=0
$$

5. Balance of forces in the GOW theory. In (3) Gurtin, Oliver and Williams suggest representations differing from the previous ones. We again shall require balance of force for all combinations of constituent bodies and therefore introduce balance Eqs. (2) and (11). The representation formulae are

$$
F_{i j}(A, C)=\int_{\partial A \cap \partial C} \sigma_{i i}{ }^{\partial A} d a
$$

for every $(A, C) \in \operatorname{sep} \mathbf{B}^{2}$ and all $i, j \in I$;

$$
F_{i i}(A, C)=\int_{A} p_{i j} d v+\int_{\partial A} \tau_{i j}{ }^{\partial A} d a+\int_{\partial A \cap C} \sigma_{i j}{ }^{\partial A} d a
$$

for every $(A, C) \in \mathbf{B}^{2}$ with $A \subseteq C$ and all $i, j \in I$ with $i \neq j$;

$$
F_{i}{ }^{\circ}(A)=\int_{A} b_{i}{ }^{*} d v+\int_{\partial A \cap \partial B} f_{i} d a
$$

for every $A \in \mathrm{B}$ and $i \in I$. The density functions ${\sigma_{i j}}^{s}$ and $\tau_{i j}{ }^{8}$ are defined on any smooth manifold $\delta$ in $B$ and map $\delta$ into $\mho_{;} f_{i}$ maps $\partial B$ into $V_{;} p_{i j}$ and $b_{i}{ }^{e}$ are as before. The interpretations of ${\sigma_{i j}}^{s}$ and $\tau_{i j}{ }^{8}$ are the same as before. ${ }^{3}$ The balance equations (2) and (11) become in this scheme:

$$
\int_{\partial A}\left({\sigma_{i I}}^{\partial A}+\tau_{i I}{ }^{\partial A}\right) d a+\int_{A}\left(p_{i}+b_{i}{ }^{\circ}\right) d v=0
$$

${ }^{3}$ The distinction between this and the previous representations is made clear by examining $F_{i j}(A, C)$ where $\bar{A} \subset C$. OW requires $F_{i j}(A, C)=\int_{A} p_{i j} d v$, while GOW requires $F_{i j}(A, C)=\int_{A} p_{i j} d v+\int_{\partial A}\left(\sigma_{i j}{ }^{\partial A}\right.$ $\left.+\tau_{i j}{ }^{\partial A}\right) d a$. GOW reduces to OW if we require, as is a consequence of the axiom scheme of OW, that $\sigma_{i j} s+\tau_{i j} s=0$ for all $S$ (cf. (15)). 
for every $A \in \mathbf{B}$ and $i \in I$;

$$
\int_{\partial A}\left(\tau_{i j}{ }^{\partial A}+\tau_{i i}{ }^{\partial A}\right) d a+\int_{A}\left(p_{i j}+p_{i i}\right) d v=0
$$

for every $A \in \mathrm{B}$ and all $i, j \in I$ with $i \neq j$;

$$
\int_{s}\left({\sigma_{i j}}^{s}+{\sigma_{i i}}^{-s}\right) d a=0
$$

for every smooth manifold $\delta$ in $B$ and all $i, j \in I$. Here

$$
\sigma_{i I}^{s}=\sum_{i \in I}{\sigma_{i i}}^{s}, \quad \tau_{i I}^{s}=\sum_{\substack{i \in I \\ j \neq i}} \tau_{i i}^{s}
$$

and $p_{i}$ is as before.

In order to ensure that the force functions are additive it is necessary that

$$
{\sigma_{i j}}^{s}+{\tau_{i j}}^{s}=-{\sigma_{i j}}^{-s}-\tau_{i j}{ }^{-s} \quad(i \neq j),
$$

for all s. From (26), (27) and (29) we may deduce the existence of stress tensor fields $T_{i i}: B \rightarrow \mathfrak{L}(\mathcal{U})$ such that

and

$$
\begin{aligned}
& \sigma_{i I}{ }^{8}+\tau_{i I}{ }^{s}=T_{i i} n \\
& \tau_{i i}{ }^{8}+\tau_{j i}{ }^{8}=-T_{i j} n,
\end{aligned}
$$

where $n$ is the unit normal to the smooth surface $\delta$. (The choice of the minus sign in $(30)_{2}$ is to make the equations parallel those of Sec. 4.) The local forms of (26), (27) and (28) are now

$$
\begin{aligned}
& \operatorname{div} T_{i i}+p_{i}+b_{i}{ }^{e}=0, \text { all } i, \\
& \operatorname{div} T_{i i}-p_{i j}-p_{i i}=0, \quad i \neq j, \\
& \sigma_{i j}{ }^{s}+\sigma_{i i}{ }^{-s}=0 .
\end{aligned}
$$

Eqs. (31) comprise the complete set of local balance equations if (29) is also required. Eqs. (31) are formally the same as (19), and indeed would reduce to (19) if it were required that ${\sigma_{i j}}^{s}=-\tau_{i j}{ }^{s}$ for all $s$. Given boundary forces $f_{i}$ on $\delta \subset \partial B$, the boundary equations for this system are

$$
\begin{aligned}
\left.T_{i i} n\right|_{s}-\sum_{\substack{j \in I \\
j \neq i}} \tau_{i j}{ }^{s}=f_{i}, \\
\left.T_{i j} n\right|_{s}+\tau_{i j}{ }^{s}+\tau_{i j}{ }^{s}=0 .
\end{aligned}
$$

Again (32) is exactly the same as (20). Thus, except for the auxiliary condition (29), this formulation yields exactly the same local equations of balance, with the same boundary conditions as the formulation in Sec. 4.

As before, there are no further equations necessary to guarantee the balance of forces, but exactly as before it is possible to formulate a global stress balance equation for the entire mixture and to introduce the stress tensors $T_{i}$ which apparently correspond to the classical stress tensors.

\section{REFERENCES}

[1] M. L. Oliver, On balanced interactions in mixtures, Arch. Rational Mech. Anal. 49, 195-224 (1972)

[2] W. O. Williams, On the thermodynamics of mixtures, Arch. Rat. Mech. Anal., to appear.

[3] M. E. Gurtin, M. L. Oliver and W. O. Williams, On balance of forces for mixlures, Quart. Appl. Math. 30, 527-530 (1973) 